\title{
Settling Analyses and Investigations of Clayey Sediments to Storage in Geotubes
}

\author{
Marco Perna ${ }^{1}$, Annamaria De Vincenzo ${ }^{2}(0)$, Giuseppe Di Nunzio ${ }^{3}$, Bruno Molino ${ }^{4}$ and Luigi Ambrosone ${ }^{1, *}(\mathbb{D})$ \\ 1 Department of Medicine and Health Sciences V. Tiberio, University of Molise, 86100 Campobasso, Italy; \\ marco.perna@unimol.it \\ 2 Engeneering School, University of Basilicata, 85100 Potenza, Italy; annamaria.devincenzo@unibas.it \\ 3 Consorzio per la Bonifica della Capitanata, Corso Roma, 2, 71121 Foggia, Italy; \\ giuseppe.dinunzio@bonificacapitanata.it \\ 4 Department of Biosciences and Territory (DIBT), University of Molise, Contrada Lappone, 86090 Pesche, Italy; \\ bruno.molino@unimol.it \\ * Correspondence: ambroson@unimol.it; Tel.: +39-0874-404715
}

check for updates

Citation: Perna, M.; De Vincenzo, A.; Di Nunzio G.; Molino, B.; Ambrosone, L. Settling Analyses and Investigations of Clayey Sediments to Storage in Geotubes. Sustainability 2021, 13, 8475. https://doi.org/ $10.3390 /$ su13158475

Academic Editor: Saeed Farrokhpay

Received: 2 June 2021

Accepted: 26 July 2021

Published: 29 July 2021

Publisher's Note: MDPI stays neutral with regard to jurisdictional claims in published maps and institutional affiliations.

Copyright: (c) 2021 by the authors. Licensee MDPI, Basel, Switzerland. This article is an open access article distributed under the terms and conditions of the Creative Commons Attribution (CC BY) license (https:// creativecommons.org/licenses/by/ $4.0 /)$.

\begin{abstract}
Understanding the sedimentation behaviour and chemical-physical properties of clay sediments is crucial in planning their storage in geotubes. Analysis of the sedimentation curves of the solids taken from the artificial reservoir of the Camastra reveal that a two-parameter curve identifies all the sedimentation profiles. One parameter depends on the type of settling material, and the other is the lifetime of the process. Using the solid concentration per unit area, the lifetime is converted into a true kinetic constant. These parameters uniquely identify the sedimentation profile to be used in the management of dredging using geotubes. Furthermore, the resulting particles after the decantation of the coarse-grained solids are dispersed according to a log-normal distribution with an average diameter between 250 and $500 \mathrm{~nm}$. The low $\zeta$-potential of the particles dispersed in the supernatant indicates that they can either aggregate or adsorb to the geotube tissue, slowing or blocking the dredging operations.
\end{abstract}

Keywords: sedimentation; structure forming; particle size

\section{Introduction}

The artificial reservoirs, which carry out the function of a garrison for the hydraulic safety of the territories, undoubtedly represent a strategic infrastructural system for every nation. Indeed, through the management of the reservoirs, the water resource indispensable for daily life is regulated [1]. This means that an alteration in water supply affects the entire economy of a country. It is therefore clear that the safeguarding and protection of water reservoirs is a problem of fundamental importance for effective sustainability of the water asset. This problem is even more serious if we keep in mind that most of the reservoirs currently functioning are obsolete and will reduce their useful capacity in the coming years. Restoring the original, useful capacity of the reservoirs means that one has to remove quantities of solid material. This, in real cases, produces unsustainable costs, both for the quantities involved and for the technical and economic difficulties associated with the de-muding operations. To overcome these difficulties, albeit partially, the concept of sustainable useful capacity was recently introduced [2]. Doing so, one has to recover not the total capacity of the reservoir but only the part designed to guarantee the water demand of the users [3]. It is therefore no longer possible to postpone the recovery of the capacity of the reservoirs together with a careful reuse planning of sediments for greater environmental sustainability. Usually, a hydrosuction technique is used to pump the watersediment mixture into suitable geotubes. However, geotubes have found wide use only for the recovery of very coarse-grained material, such as marine material $[4,5]$, but only rarely for the recovery of clayey materials from lakes [6,7]. Indeed, in addition to storage, alternative solutions for these materials are being studied $[8,9]$. 
In very diluted systems, the number of particles is very small, and they can only grow to a limited extent. When the initial particle concentration is high enough, continuous structures form through the whole bulk [10]. The operations of collecting clayey sediments in geotubes foresee the alternation of filling and dewatering phases. Accordingly, the small dispersed particles undergo dilution and enrichment cycles, where three-dimensional structures are formed that reduce the texture of the geotube by slowing down the dewatering phase. Furthermore, if the structures are very large, there is a risk of completely inactivating the geotube function. Little work has been done to study the effects of fine clay suspensions on geotube function due to a lack of experimental data. This research aims to determine some chemical-physical parameters of fine clay suspensions taken from an Italian reservoir (Camastra, Potenza). Furthermore, the properties of the sedimentation process are also studied in order to extract useful information for a correct planning and management of the reservoir removal operations [11,12].

\section{Materials and Methods}

\subsection{Materials}

Samplings of sediment in different points of Camastra reservoir (Potenza, Italy) was accomplished. As it can be seen in the map of Figure 1, the first sample of material is representative of the area adjacent to the water intake tower, where some outlets are blocked due to silting. This sample, hereafter, is referred to as (PI).

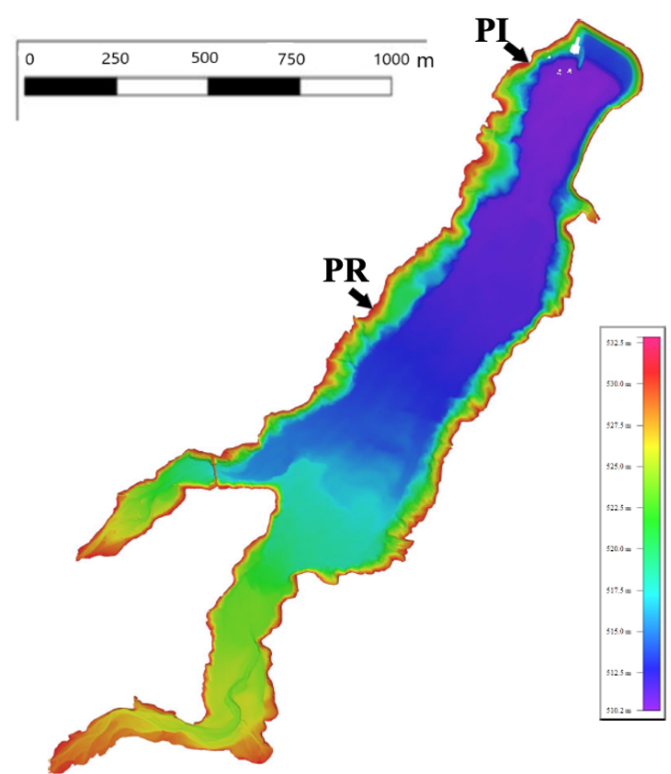

Figure 1. Map of the Camastra reservoir showing the sediment sampling points.

The second sampling was done upstream at a point towards the centre of the dam, on the access road to the reservoir (see Figure 1). This sample, hereafter, is referred to as (PR). The bathymetric surveys revealed an extensive silting up from the reservoir at this altitude. Anyhow, both sampling points, for long periods of the year, are below the maximum water level of the dam. Chemical analyses on both samples confirm the sediment to be non-hazardous waste with similar molecular composition. In particular, the material is clay rich in $\mathrm{Al}_{2} \mathrm{O}_{3}(14.3 \%)$ and $\mathrm{Fe}_{2} \mathrm{O}_{3}(7.9 \%)$.

\subsection{Preparation of Samples}

The samples were laid out in large jars and continually turned over to allow the solid to dry. This drying phase of the material lasted about 2 months, at the end of which the dry solid was homogenized in a mortar and stored in a closed container at room temperature. 


\subsection{Density Measurements}

Density measurements were carried out with a graduated cylinder, carefully weighed and calibrated with distilled water. For each sample, 7 measurements were performed, the average density values were $\bar{\rho}=1.44 \pm 0.05$ for the PI sample and $\bar{\rho}=1.45 \pm 0.02$ for the PR sample.

\subsection{Batch Settling Tests}

Glass cylinders 25, 50, 100, 250, 500 and $1000 \mathrm{~cm}^{3}$ were used for containing the suspensions. They were graduated in $1 \mathrm{~cm}^{3}$ divisions and were kept vertical. During the measurement, the cylinder was illuminated with a lamp, and the solids/liquid interface (mud line) was carefully monitored and recorded as a function of settling time. Photographs were also taken of the settling suspensions periodically. All suspensions investigated contained $10 \%(v / v)$ of solid.

In order to understand the conditions under which structure formation takes place, the concentration of the disperse phase is most usefully defined in terms of the volume fraction of the disperse phase, $\Phi$. Structured systems only arise if $\Phi$ is greater than some critical value, $\Phi_{\text {crit }}$. Because the density of the solid particles is significantly different from that of water, this parameter can be experimentally measured as the relative volume of sediments

$$
V_{s}=\frac{\text { volume of sediment }}{\text { volume of the disperse phase in the sediment }}
$$

Technically, this is easily done by dispersing a known volume of sample in water and measuring the equilibrium volume. The criterion for estimating a structure formation is

$$
\Phi \geq \Phi_{\text {crit }}=\frac{1}{V_{s}}
$$

Sediment volumes provide valuable information on the nature of the structure. For instance, agglomerates form soft and loosely packed sediments and have large sediment volumes that are easily re-dispersed in most cases; however, stable suspensions will form sediments of smaller volume that are not easily re-dispersed, as schematically displayed in Figure 2 .

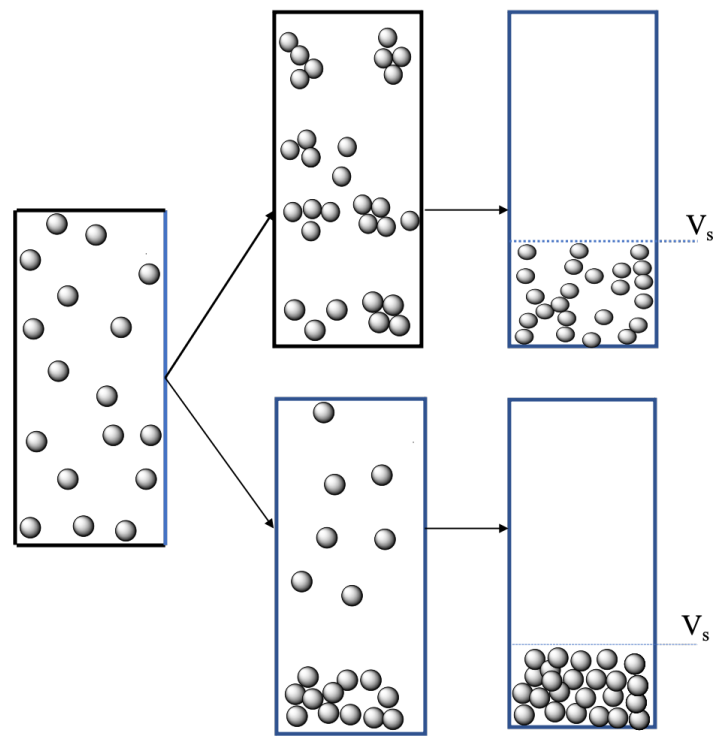

Figure 2. Sedimentation of dispersions forming compact or loose structures.

\subsection{Dynamic Light Scattering and $\zeta$-Potential}

Zetasizer Nano ZS90, Malvern Instruments Ltd., Worcester-shire, UK, was used to measure particle size at a 90-degree scattering angle using Dynamic Light Scattering (DLS). 
The DLS setup used was equipped with an Multiple-Digital-Correlator and a $50 \mathrm{~mW}$ $\mathrm{He} / \mathrm{Ne}(\lambda=633 \mathrm{~nm})$. In DLS experiments, the temporal fluctuations of the scattered intensity due to Brownian motion in the sample were analyzed by an appropriate software that evaluates the size particles in the range $0.3 \mathrm{~nm}-5 \mu \mathrm{m}$. The average hydrodynamical diameter of particles was calculated from at least three measurements. The DLS setup was also equipped to measure the $\zeta$-potential and electrophoretic mobility using laser Doppler micro-electrophoresis. In particular, the electrophoretic mobility of a large enough number of single particles was measured and converted to $\zeta$-potential unbiased by the size distribution.

\subsection{DSC-TG Measurements}

The thermal analysis equipment consisted of a DSC-TG unit, TA Instruments Model Q600. An empty platinum crucible served as a reference. A heating rate of $20^{\circ} \mathrm{C} \cdot \mathrm{min}^{-1}$ was used during all the experiments. The samples were analyzed in dry air at $100 \mathrm{~cm}^{3} \cdot \mathrm{min}^{-1}$ in the temperature range $20-900{ }^{\circ} \mathrm{C}$. The sample mass was around $25-30 \mathrm{mg}$ in all the experiments. The heat flow was measured as a function of temperature, while the sample was exposed to a controlled temperature program.

\section{Results and Discussion}

TG curves of samples PI and PR subject to gradual heating are displayed in Figure 3, as well as their derivative (DTG) [13]. Both samples exhibit similar patterns, indicating that they have identical chemical composition but different geometric shape and particle size. The mass loss curve exhibits a decreasing trend, and DTG plots show peaks in correspondence with the points of maximum mass loss rate. Both the two inflections in the TG curve and the two peaks exhibited by the DTG plot reveals that the material degradation takes place in a two-stage process. The first stage, beginning at room temperature and ending around $180^{\circ} \mathrm{C}$, is due to the loss of water not chemically bound to the sample.

This degradation step corresponds to a mass loss of $1.5 \%$ for the sample and $2 \%$ for sample 2. The second stage involves the largest loss in mass of the samples, starting from $180{ }^{\circ} \mathrm{C}$ and ending at $800-820^{\circ} \mathrm{C}$, due to both the organic part degradation of the material and the loss of chemically bound water. This step represents the mass loss of $15.5 \%$ of the PI sample and $24 \%$ of the PR sample. These results are in excellent agreement with the values measured on clay [14]. DSC measurements are displayed in Figure 3. The thermal curves of both samples are characterized by two large endothermic peaks. The first peak is centred around $180^{\circ} \mathrm{C}$ and the second, $800{ }^{\circ} \mathrm{C}$, for both samples. They correspond to dehydration and volatilization processes.

\section{Settling Measurements}

Volumes of 25, 50, 100, 250, 500 and $1000 \mathrm{~cm}^{3}$ containing 10\% $(v / v)$ solids were subjected to the settling test. For PI sample, $\Phi_{\text {crit }}=0.68 \pm 0.04$ was found, while for the PR sample, $\Phi_{\text {crit }}=0.71 \pm 0.02$. It is clear that both solid samples exhibit the same value of $\Phi_{\text {crit }}$, again indicating that their chemical composition is essentially identical. It is interesting to note that these results agree well with known properties of a random sphere where the volume fraction of dense packings is 0.64 . The clarity of the supernatant is an important factor; therefore, the turbidity of the supernatant after flocculation was also monitored as a criterion for evaluating the ability to form a solid-like spatial structure. The clarity of the supernatant is an important factor, and the turbidity of the supernatant after flocculation was monitored as a criterion for evaluating the ability to form a solid-like spatial structure. Such parameter was evaluated by the particle size distribution measured through DLS; a technique currently used for measuring particle size over a size range from nanometres to micrometres [15]. Typical number-weighted particle size distributions obtained for PI and PR samples are shown in Figure 4. First, it should be noted that the size measured via the DLS technique is the hydrodynamic diameter of a theoretical sphere diffusing with the same speed as the measured particle. Accordingly, the size measured 
with this technique is bigger in comparison with the volume of naked particle. This effect is the consequence of the thin liquid layers between the particles. However, the difference between the hydrodynamic diameter and the particle's own diameter is generally small. Typical number-weighted particle size distributions obtained for PI and PR samples are displayed in Figure 4. As it can be seen, both samples exhibit a log-normal distribution with a geometric mean of diameters of $350 \pm 5 \mathrm{~nm}$ for the PI sample and $250 \pm 4 \mathrm{~nm}$ for the PR sample.
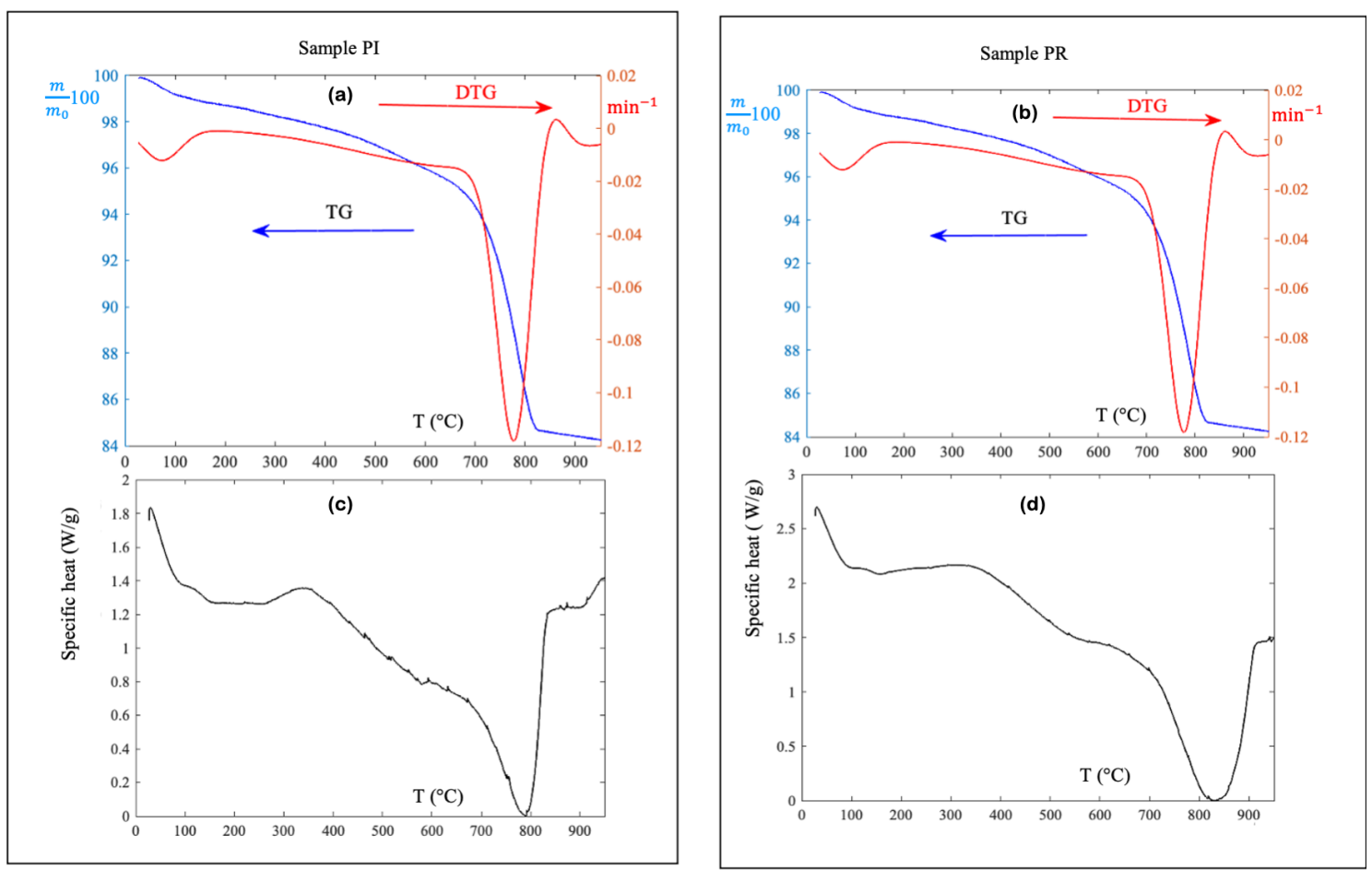

Figure 3. TG and DTG curves of (a) PI sample and (b) PR sample, $m_{0}$ being the initial mass of material. DSC traces of (c) PI sample and (d) PR sample. Measurements were performed under dry air stream $\left(100 \mathrm{~cm}^{3} \mathrm{~min}^{-1}\right)$ and a rate heating of $20{ }^{\circ} \mathrm{C} \min ^{-1}$.

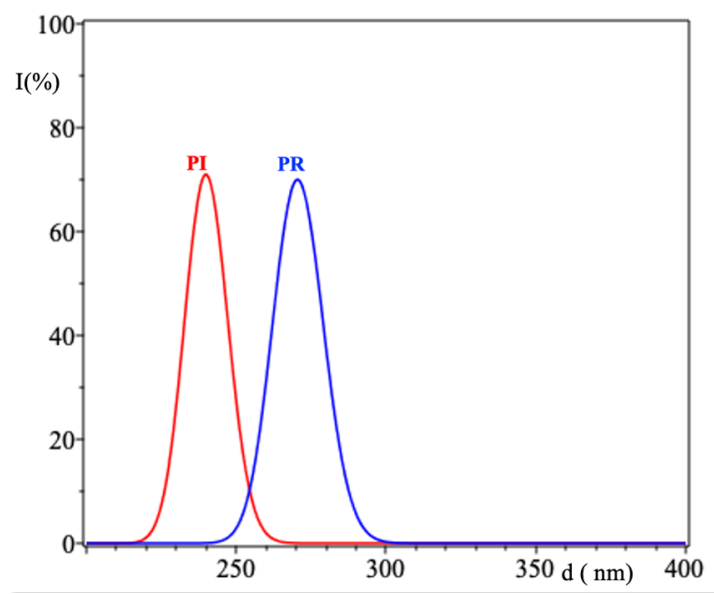

Figure 4. Typical intensity (I) size distribution, a number-weighted size distribution obtained by DLS measurements on supernatant after sedimentation for two solid samples taken from the Camastra (Italy) reservoir. The symmetry on a logarithmic scale implies that the distribution is log-normal. 
The supernatant is nothing more than a very diluted dispersion so that the sedimentation rate can be established with Stokes' law:

$$
u_{s}=\frac{d^{2}\left(\rho_{s}-\rho_{w}\right) g}{18 \eta}
$$

where $\rho_{s}$ and $\rho_{w}$ are densities of dispersed phase and water, respectively, $\mathrm{d}$ is the particle diameter and $\eta$ the viscosity of the medium (water). Using $d$ measured via DLS, it is easy to calculate $u_{s}=1.4 \cdot 10^{-8} \mathrm{~ms}^{-1}$ for sample PI and $u_{s}=2.1 \cdot 10^{-8} \mathrm{~ms}^{-1}$ for PR. These extremely low values mean that the particles remain in suspension for very long times. Furthermore, the $\mathrm{pH}=7$ remained constant throughout the process, indicating that solids do not release specific ions. In order to estimate the physical behaviour of these particles, the $\zeta$-potential of the suspension was measured. Both samples exhibit equal $\zeta$-potential, specifically one measure $\zeta=-10 \pm 1 \mathrm{mV}$ and $\zeta=-11 \pm 2 \mathrm{mV}$ for PI and PR, respectively. The $\mathrm{pH}$ determinations carried out before and after the $\zeta$-potential measurements oscillate from 6.8 to 7.2; therefore, the $\mathrm{pH}$ can be assumed to be constant. Such low values of $\zeta$ potential are indicative of an ability to aggregate and form a particle structure. On the other hand, geotubes are generally made of polypropylene, so the solid particles can adsorb on the polymer and aggregate, preventing water from escaping. This reduces the effectiveness of a geotube.

Hydrophobic surfaces tend to be rejected from the water structure. This effect promotes coagulation between particles. Very loose structures are obtained if only parts of the surface of the particles are hydrophobic.

In order to quantitatively evaluate the sediment accumulation rate, we introduce the normalized sediment volume as follows

$$
\mathcal{V}_{s}(t)=\frac{V(t)-V_{0}}{V_{\infty}-V_{0}}
$$

where $V(t)$ is the volume of solids measured at the solid/liquid interface at time $t, V_{0}$ the initial dispersion volume and $V_{\infty}$ the sediment volume at equilibrium. In Figure $5, \mathcal{V}_{s}$ vs. $t$ is shown for both PI and PR samples. Obviously, the PR sample, being made up of coarser-grained solids, settles more rapidly. Nevertheless, the shape of the curves is similar for both sample sample types. The shape of these curves was determined by the non-linear fitting to experimental data and turned out to be

$$
\mathcal{V}_{s}=\tanh \left(\frac{t}{\tau}\right)^{\sigma}
$$

where $\tanh$ is the hyperbolic tangent, and $\tau$ and $\sigma$ are two adjustable parameters. While the value of $\sigma$ remains constant for all samples, i.e., $\sigma=1.8 \pm 0.3$ for the PI sample and $\sigma=1.6 \pm 0.2$ for the PR sample), $\tau$ varies significantly as a function of the initial dispersion volume. The fact that $\sigma \approx 2$ implies that the growth rate of $\mathcal{V}_{s}$ is initially slow, then increases. This suggests that there is some factor of impedance that affects particles fall. Although this may be ascribed to a certain amount of interference between particles, it is also possible to ascribe it, at least in part, to the displacement of the fluid by the downward movement of particles. Thus, the value of $\sigma$ is fixed by the kind of solid material.

As regards the parameter $\tau$, it represents a mean lifetime [16], i.e., an average of the time with which the solid accumulates during the sedimentation process. Figure 6 shows $\tau$ as a function of the initial dispersion volume for PI and PR samples. 

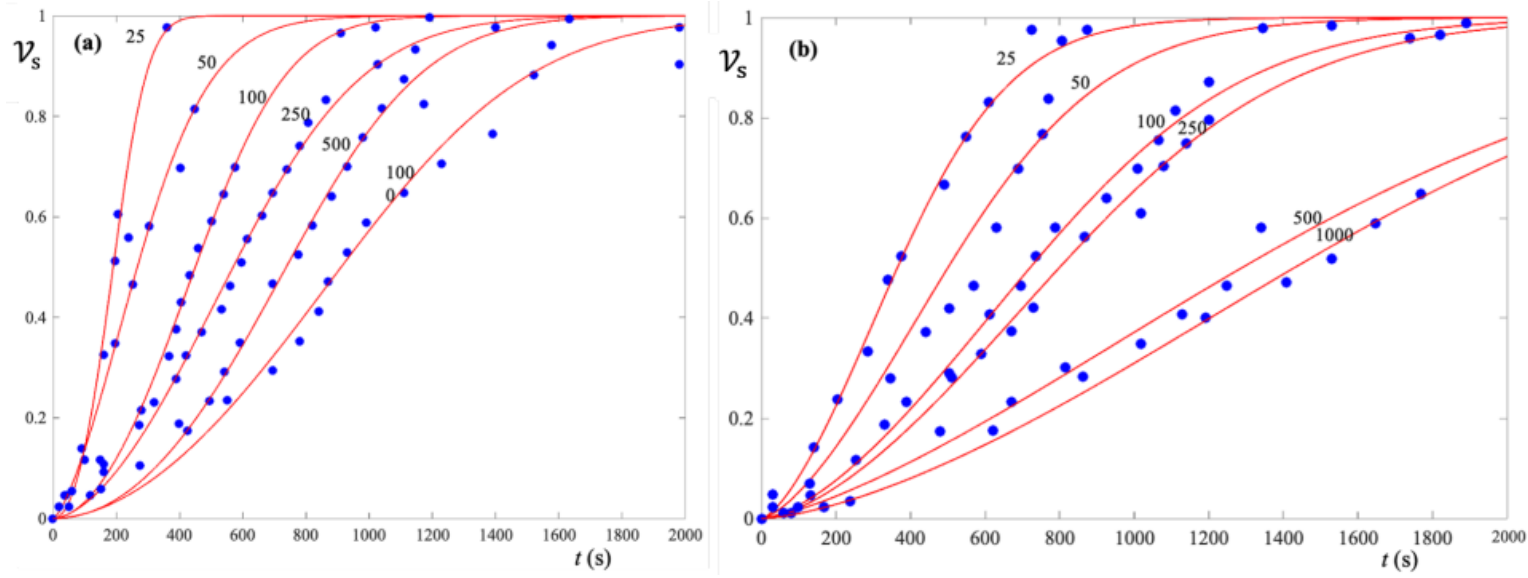

Figure 5. Normalized volume of sediments as a function of time for the (a) PI sample and (b) PR sample. Numbers on each curve represent the initial volume $\left(\right.$ in $\mathrm{cm}^{3}$ ) used for sedimentation experiments.
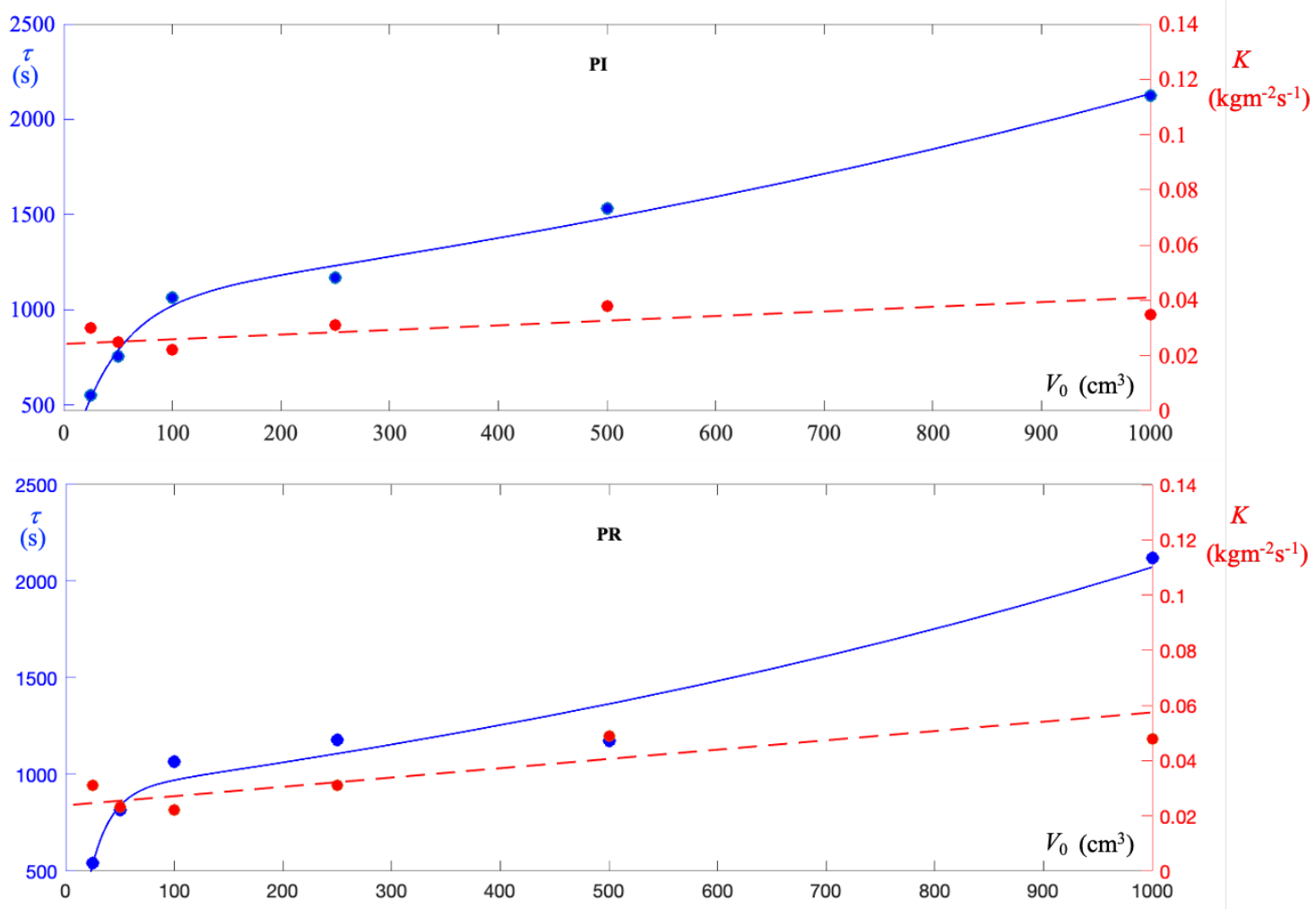

Figure 6. Lifetime as a function of the initial volume of sedimentation at constant volume fraction of solids (10\%). If the concentration per unit area is taken into account, the lifetime is converted into a true kinetic constant, $K$.

In order to explain the observed variation in $\tau$, we note that in cylinders of unequal depths filled with the same kind of solid and at the same concentration, the solid in the shallow cylinder begins to compress more quickly, indicating that thickening is a function of time. This implies that the intrinsic rate of sedimentation $\frac{1}{\tau}$ is not a true constant but rather depends on the concentration of solid per unit area

$$
\frac{1}{\tau}=\frac{K}{C_{0} H_{0}}
$$

where is the initial concentration $\left(\mathrm{kgm}^{-3}\right), H_{0}$ is the initial height of solids and $\mathrm{K}$ is the true kinetic constant of sedimentation. 
Results shown in Figure 6 (red lines) indicate, within the experimental error, that $K=0.034 \pm 0.02 \mathrm{kgm}^{-2} \mathrm{~s}^{-1}$ for PI and $K=0.038 \pm 0.05 \mathrm{kgm}^{-2} \mathrm{~s}^{-1}$ remain practically constant. This means that, knowing the geometric parameters of the geotube from the value of $K$, it is possible to determine $\tau$ for the particular process of mud removal. Furthermore, from the investigation of the material extracted from the reservoir, $\sigma$ is obtained, so that the pair $(\sigma, \tau)$ allows to determine the sediment volume for a specific reservoir.

Moreover, the solid concentration, $C(t)$, is related to the volume by a simple mass balance of solids

$$
V(t)=\frac{C_{0} V_{0}}{C(t)}
$$

Equation (7) is valid during all of the sedimentation, including the initial and final instants. Applying Equation (7) to the terms $V, V_{0}, V_{\infty}$ in Equation (4) and simplifying the resulting equation gives

$$
\frac{1}{C(t)}=\frac{1}{C_{0}}+\left(\frac{1}{C_{0}}-\frac{1}{C_{\infty}}\right) \tanh \left(\frac{t}{\tau}\right)^{\sigma}
$$

Thus, the experimental results allow determining the parameters $(\sigma, \tau)$ sufficient to generate the sedimentation profile of the material and concentration of solids. In other words, the necessary equations are obtained for programming and managing the dredging process of a reservoir using geotubes. Obviously, this point must be further investigated by extending the investigation to different solid materials. However, some general aspects can be pointed out.

The equations discussed on the basis of laboratory results provide the reservoir manager with a useful tool to control sediment formation. Through the knowledge of the sediment formation rate, the manager can appropriately control and maintain a useful capacity of the reservoir in order to satisfy all the water requirements of the territory.

\section{Conclusions}

The sedimentation behaviour of clayey materials taken from the Camastra reservoir was analyzed. After sedimentation of the coarser solids, particles of $250-500 \mathrm{~nm}$ in diameter remain dispersed according to a log-normal distribution. Their z-potential is small enough to have good aggregation capacity. However, if these materials are to be stored in geotubes made of polypropylene, they could adsorb on the meshes of the fabric, slowing down (or blocking) the removal process. The analysis of the sedimentation curves reveals that a two-parameter curve, $(\sigma, \tau)$, describes the experimental results very well. The $\sigma$ parameter is constant and depends only on the kind of solid material. On the contrary, the parameter $\tau$ varies significantly with the initial volume of dispersion. However, if the solid concentration per unit area is taken into account, the lifetime parameter is converted into a true sedimentation kinetic constant, $K$, which remains practically constant for all experiments. The PR sample is taken from points distant from the reservoir and therefore consists of coarser solids. The slight change detectable in Figure 6 is due to the non-homogeneity of the solid material.

These observations provide useful information for the design and planning of the dredging process of the reservoir using geotubes. Indeed, if the geometry of the geotube and its feed rate are known, one can calculate the $\tau$ parameter directly. Then, from the characteristics of the solids, it gets the value $\sigma$ so that it can generate the sedimentation profile useful for programming dredging times. Finally, from the mass balance on the solid, one calculates the concentration of solids in the geotube, and thus, the filling process of the geotube can be managed.

Obviously, this investigation constitutes only a starting point to indicate that it is possible to find, on the basis of experimental results, management equations that allow to accurately monitor the useful capacity of a reservoir. When a sufficient amount of data is 
available, they can be grouped into convenient equations, and the method can be extended to the management of reservoirs.

Author Contributions: Conceptualization, methodology, L.A.; formal analysis, G.D.N.; investigation, A.D.V., B.M.; data curation, M.P.; writing—original draft preparation, B.M., A.D.V.; project administration, L.A., B.M., A.D.V. All authors have read and agreed to the published version of the manuscript.

Funding: This research received no external funding.

Acknowledgments: The authors thank Giuseppe D'Amato for the IT support during the drafting of the paper.

Conflicts of Interest: The authors declare no conflict of interest.

\section{References}

1. Asmadi, A.; El-Shafie, A.; Razali, S.F.M.; Mohamad, Z.S. Reservoir optimization in water resources: A review. Water Resour. Manag. 2014, 28, 3391-3405. [CrossRef]

2. De Vincenzo, A.; Molino, B.; Viparelli, R.; Caramuscio, P. A methodological approach for estimating turbidity in a river. Int. J. Sed. Res. 2011, 26, 112-119. [CrossRef]

3. De Vincenzo, A.; Molino, A.J.; Molino, B.; Scorpio, V. Reservoir rehabilitation: The new methodological approach of Economic Environmental Defence. Int. J. Sed. Res. 2017, 32, 288-294. [CrossRef]

4. Sheehan, C.; Harrington, J. An environmental and economic analysis for geotube coastal structures retaining dredge material Resour. Conserv. Recycl. 2012, 61, 91-102. [CrossRef]

5. Parab, S.R.; Chodankar, D.S.; Shirgaunkar, R.M.; Fernandes, M.; Parab, A.B.; Aldonkar, S.S.; Savoikar, P.P. Geotubes for beach erosion control in Goa. Int. J. Earth Sci. Eng 2011, 4, 1013-1016.

6. Molino, B.; Bufalo, G.; De Vincenzo, A.; Ambrosone, L. Semiempirical Model for Assessing Dewatering Process by Flocculation of Dredged Sludge in an Artificial Reservoir. Appl. Sci. 2020, 10, 3051. [CrossRef]

7. Covelli, C.; Cimorelli, L.; Pagliuca, D.; Danila, N.; Molino, B.; Pianese, D. Assessment of erosion in river basins: A distributed model to estimate the sediment production over watersheds by a 3-Dimensional LS Factor in RUSLE Model. Hydrology 2020, 7, 13. [CrossRef]

8. Messina, F.; Ferone, C.; Molino, A.J.; Roviello, G.; Colangelo, F.; Molino, B.; Cioffi, R. Synergistic recycling of calcined clayey sediments and water potabilization sludge as geopolymer precursors: Upscaling from binders to precast paving cement-free bricks. Construc. Build. Mat. 2017, 133, 14-26. [CrossRef]

9. Molino, B.; De Vincenzo, A.; Ferone, C.; Messina, F.; Colangelo, F.; Cioffi, R. Recycling of clay sediments for geopolymer binder production. A new perspective for reservoir management in the framework of Italian legislation: The Occhito reservoir case study. Materials 2014, 8, 5603-5616. [CrossRef] [PubMed]

10. Yastrebinskii, R.N.; Cherkashina, N.I.; Doroganov, V.A.; Yastrebinskaya, A.V. Effect of additives on dispersed system structure formation. Refract. Ind. Ceram. 2020, 10, 562-564.

11. Bagarani, M.; De Vincenzo, A.; Ievoli, C.; Molino, B. The Reuse of Sediments Dredged from Artificial Reservoirs for Beach Nourishment: Technical and Economic Feasibility. Sustainability 2020, 12, 6820. [CrossRef]

12. Cimorelli, L.; Covelli, C.; De Vincenzo, A.; Pianese, D.; Molino, B. Sedimentation in reservoirs: Evaluation of return periods related to operational failures of water supply reservoirs with Monte Carlo simulation. J. Water Resour. Plan. Manag. 2021, 147, 04020096 [CrossRef]

13. Bufalo, G.; Costagliola, C.; Mosca, M.; Ambrosone, L. Thermal analysis of milling products and its implications in self-ignition. J. Therm. Anal. Calorim. 2014, 115, 1989-1998. [CrossRef]

14. Yuan, P.; Tan, D.; Annabi-Bergaya, F.; Yan, W.; Liu, D.; Liu, Z. From platy kaolinite to aluminosilicate nanoroll via one-step delamination of kaolinite: Effect of the temperature of intercalation. Appl. Clay Sci. 2013, 83, 68-76. [CrossRef]

15. Stetefeld, J.; McKenna, S.A.; Patel, T.R. Dynamic light scattering: A practical guide and applications in biomedical sciences. Biophys. Rev. 2016, 8, 409-427. [CrossRef] [PubMed]

16. Venditti, F.; Bufalo, G.; Lopez, F.; Ambrosone, L. Pollutants adsorption from aqueous solutions: The role of the mean lifetime. Chem. Eng. Sci. 2011, 23, 5922-5929. [CrossRef] 